Rev. Elev. Med. vét. Pays trop., 1968, 21, 3 (387-403)

\title{
Observations sur un élevage de Glossina tachinoides West., après adoption du lapin comme animal-hôte
}

\author{
por J. ITARD, L. MAILLOT, J. BRUNET et M. GIRET
}

\begin{abstract}
RÉSUMÉ
L'adoption du lapin comme animal hôte et la réception d'un important lot de pupes ont permis d'obtenır, depuis le début de l'année 1967, un élevage florissant de Gl. tochinoides.

Malgré une intoxication accidentelle par insecticide, qui a faif tomber les effectifs à moins de 400 femelies/jour, ceux-ci dépassarent 6 mois plus tard 2.300 femelles. La langévité maximum des femelles a été de 210 jours et la production de pupes a atteint 695 pupes pour 100 femelles.

La technique utilisée est susceptıble d'être appliquée à un élevage en masse en vue de la production de mâles síeriles.
\end{abstract}

\section{I. - INTRODUCTION}

Dans un article paru en 1966 ITARD et MAILLOT ont décrit les premiers essais d'un élevage de Glossina tachinoides à partır de deux lots de pupes en provenance de la région de Fort-Lamy (République du Tchad), reçus à Maısons-Alfort les 2 et 20 avril 1965.

Les résultats obtenus furent relativement médiocres et les auteurs estimaient, à l'époque, que les conditions d'élevage convenant à $G$. morsitans étaient moins favorables à Gl. tochinoides ef que l'alımentation sur cobaye ne semblait pas indiquée pour cette dernière espèce.

Malgré une nouvelle expédition de pupes en février 1966 et l'utilisation, à partir de mars 1966 , de la poule comme anımal-hôte, l'effectıf de la souche de Gl. tachinoides est resté très faible jusque vers la fin de l'année 1966.

C'est alors que deux événements presque simultanés ont totalement modifié le comportement de cet élevage.

A la suite de la parution de l'article de $\mathrm{NASH}$, JORDAN et BOYLE (1966), décrivant la tech- nique de nourriture de $\mathrm{Gl}$. ousteni sur oreilles de lapıns, ce procédé fut appliqué, en novembre 1966, à l'élevage de Gl. tachinordes.

Au même moment nous recevions deux lots de pupes de $G$ l. tachinordes en provenance de Fort-Lamy. Le premier lot, reçu le 5 novembre 1966, comprenait 150 pupes dont sont éclos 39 mâles et 43 femelles (pourcentage d'éclosion : 54,6 p. 100). Le deuxième lot, reçu le 15 décembre 1966, comprenait 3.162 pupes qui ont donné 657 mâles et 858 femelles (pourcentage d'éclosion : 47,9 p. 100).

Malgré une intoxication accidentelle par insecticide en juin 1967, les effectifs ont alors progressé de façon remarquable et atteignaient une moyenne de 2.326 femelles par jour pour la période du 20 décembre 1967 au 18 janvier 1968, en dépit de l'utilisation, à des fins expérimentales, de 660 femelles et 3.128 pupes entre le 20 novembre 1967 et le 18 janvier 1968.

\section{II. - TECHNIQUE D'ÉLEVAGE}

Les conditions de température, d'humidité ef 
d'éclairement sont à peu près identiques à celles décrites dans l'article d'ITARD et MAILLOT.

Les imagos sont maintenus dans une pièce (dénommée « grande salle»), à une température de $25^{\circ} \mathrm{C}$ et une humidité relative d'environ 75 p. 100. Les pupes et les mouches de moins de 10 jours sont stockées dans une deuxième pièce, ou «petite salle », séparée de la première par un sas, d̀ $25^{\circ} \mathrm{C}$ et entre 85 et 90 p. 100 d'humidité relative.

Les pupes, récoltées chaque matin, sont placées dans des tubes de Borrel stériles, sans sable, à raison de 30 d̀ 35 pupes par tube.

Les femelles sont accouplées à l'âge de 3 à 4 jours avec des mâles âgés de 7 à 10 jours. Après la période de réunion des sexes, qui dure de 4 à 6 jours, les femelles sont séparées des mâles et placées, par groupes de 15 à 20 mouches dans des cages de type ROUBAUD, constituées par une armature métallique plastifiée de $14 \times 8,5 \times 5 \mathrm{~cm}$, recouverte d'une housse en tulle de tergal à mailles carrées de $2 \mathrm{~mm}$ de côté. Ces dimensions permettent aux larves pondues de passer à travers la maille et de puper au fond de la cuvette où sont placées les cages.

Les cages contenant les femelles, groupées par dates d'éclosion, sont placées, par lots de 8 à 10 cages, dans des cuvettes rectangulaires en poly-éthylène de $34 \times 26 \times 6,5 \mathrm{~cm}$, et reposent sur des chevalets constitués par deux baguettes de verre réunıes par un tube souple en soreflon, de telle sorte que l'espace compris entre les cages et le fond de la cuvette soit d'environ 1,5 cm. Le fond de la cuvette est en outre garni d'une feuille de papier Joseph qui absorbe les déjections des mouches et sous laquelle les larves se glissent pour se transformer en pupe.

Chaque cuvette contient ainsi 150 femelles environ dont l'âge, au moment de la séparation, est compris entre 7 et 10 jours.

Ces modifications dans la technique d'élevage nous ont été imposées par l'accroissement des effectıfs des différentes souches. A titre d'exemple, les effectifs journalıers, pour la période du 20 décembre 1967 au 18 janvier 1968, comprenaient 2.326 femeiles de Gl. tachinoides, 2.352 femelles de Gl. morsitans morsitans, 179 femelles de Gl. austeni, et environ 1.000 mâles des trois espèces. Nous nous limitons actuellement d̀ des effectifs de 2.000 femelles environ par jour ef par espèce, tout le surplus étant utilisé à des fins expérimentales.

Les mouches sont nourries, chaque jour, sur les oreilles du lapin. Nous avons suivi la technique décrite par $\mathrm{NASH}$, quant au mode de fixation des cages sur les oreilles du lapin et à la contention de celui-ci. Nous utilisons 6 lapins par jour pour nourrir la totalıté de nos mouches. Chaque lapin est utilisé pendant une semaine, puis reste au repos pendant trois semaines, ce qui nécessite le maintien d'un effectif total de 24 lapıns.

Nous nous sommes servis, dans les premiers temps, de lapins de race «Bélier français», caractérisés par leurs oreilles pendantes et longues d'environ 15 à $20 \mathrm{~cm}$. Ces lapins, qui sont obtenus par sélection et croisements consanguins, sont très peu rustiques et d'un prix d'achat élevé. Aussi leurs préférons-nous actuellement des lapins adultes de race Bouscat, pesant de 5 à 7 kgs, dont les oreilles mesurent une quinzaine de centimètres. Ces animaux nous donnent d'aussi bons résultats que les Béliers français.

Les cages ne sont maintenues sur l'oreille $d u$ lapin que pendant 3 à 4 mınutes. Nous n'incitons pas les mouches à se nourrir et celles qui n'ont pas pris leur repas de sang ne peuvent se nourrir que le lendemain. En outre, les mouches ne sont plus nourries le dimanche, depuis le 24 décembre 1967. En ne maintenant les cages que 3 à 4 minutes sur l'oreille du lapin, la totalité des effectifs sont nourris en une matinée.

Les pupes produites depuis la veille sont récoltées chaque matin, les femelles mortes dénombrées, les mouches écloses réparties dans des cages correspondant à leur sexe ef leur espèce.

Tous les renseignements obtenus sont consignés sur fiches (fig. 1, 2 et 3 ).

On effectue en outre, chaque mois, l'inventaire des femelles, de façon d corriger éventuellement les erreurs ou omissions qui auraient pu se produire lors de la transcription des résultats journaliers.

\section{III. - RÉSULTATS}

\section{A. - Comportement général de l'élevage.}

Le graphique 1 résume les résultats obtenus au cours de l'année 1967. 


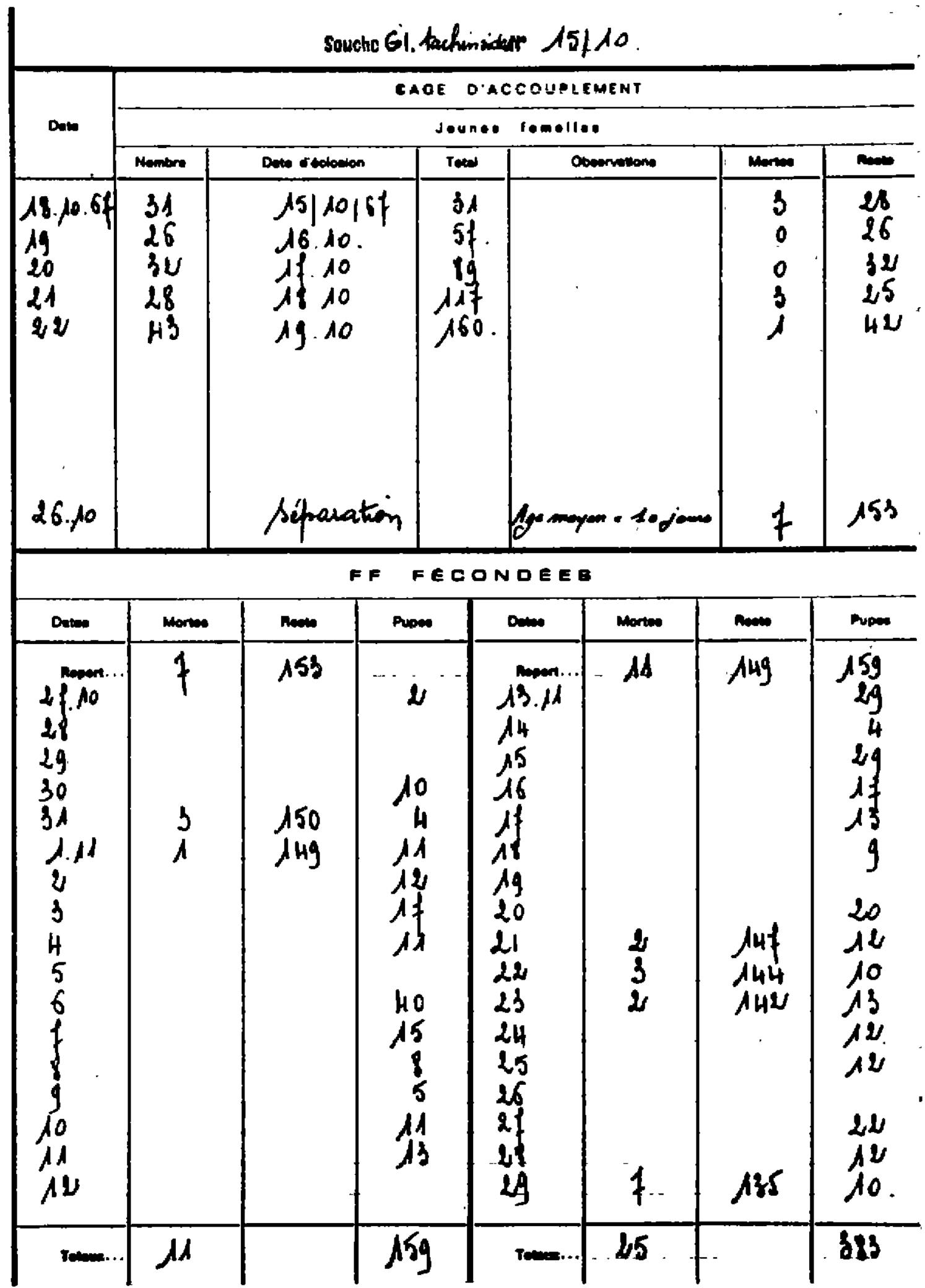

Fig. 1. - Fiche d'un lot de fernelies accouplées. 
Finto

PUPES Souctickl.kehinoides

\begin{tabular}{|c|c|c|c|c|c|c|c|c|c|c|c|}
\hline Dom nom & Nombore do & octomonon & Fomeilus & Manes & NE & Don ono & Nombere do & $\begin{array}{l}\text { Oole of } \\
\text { oclosion }\end{array}$ & Femeleter & Male: & me \\
\hline $30 / 1$ & 133 & $\begin{array}{l}262 \\
2 f .2 \\
212 \\
29.2 \\
1.3 \\
2.3\end{array}$ & $\begin{array}{l}\text { if } \\
20 \\
20 \\
4\end{array}$ & $\begin{array}{c}6 \\
29 \\
2 b \\
1 \\
4\end{array}$ & 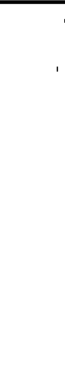 & 3 시사 & 105 & $\begin{array}{r}2 f . U \\
2.0 \\
29.8 \\
7.3 \\
2.3 \\
H .3\end{array}$ & $\begin{array}{l}18 \\
18 \\
10\end{array}$ & $\begin{array}{l}1 \\
8 \\
1 \\
1 \\
20 \\
9 \\
3\end{array}$ & \\
\hline \multicolumn{3}{|c|}{ Taneme } & 61 & fe & 10 & \multicolumn{3}{|c|}{ Tetoum. } & $8 h$ & $5 g$ & $1 U$ \\
\hline$\left.1\right|^{\nu}$ & 166 & $\begin{array}{l}28.2 \\
\text { lg. } \\
1.3 \\
1.3 \\
4.3 \\
5.3\end{array}$ & $\begin{array}{r}9 \\
27 \\
26 \\
20 \\
1\end{array}$ & $\begin{array}{r}51 \\
H\end{array}$ & 9 & $2 / 2$ & 208 & $\begin{array}{l}29.8 \\
1.3 \\
2.3 \\
4.3 \\
5.3 \\
6.3\end{array}$ & $\begin{array}{l}8 \\
28 \\
31\end{array}$ & $\begin{array}{c}61 \\
3 A \\
A \\
A 2\end{array}$ & 13 \\
\hline \multicolumn{3}{|c|}{ Toneve } & 83 & $f_{4}$ & $g$ & \multicolumn{3}{|c|}{ 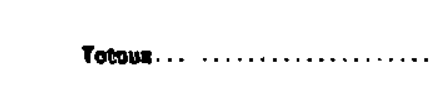 } & 89 & 106 & 13 \\
\hline $3 / 2$ & 211 & $\begin{array}{l}2.3 \\
4.3 \\
5.3 \\
6.3 \\
1.3\end{array}$ & $\begin{array}{r}6 \\
49 \\
31 \\
6\end{array}$ & $\begin{array}{c}1 \\
23 \\
53 \\
13 \\
3 \\
4\end{array}$ & Af & $5 \mid y$ & 344 & $\begin{array}{r}2.3 \\
4.3 \\
5.3 \\
6.3 \\
3.3 \\
1.3 \\
11.3\end{array}$ & $\begin{array}{l}11 \\
63 \\
60 \\
44 \\
3 \\
y\end{array}$ & $\begin{array}{l}10 \\
45 \\
54 \\
36 \\
1 \\
4\end{array}$ & 14 \\
\hline & & & gd & 103 & Af & בחמד & & & 186 & Aluy & 14 \\
\hline
\end{tabular}

Fig. 2, - Fiche de production et d'éclosion des pupes. 


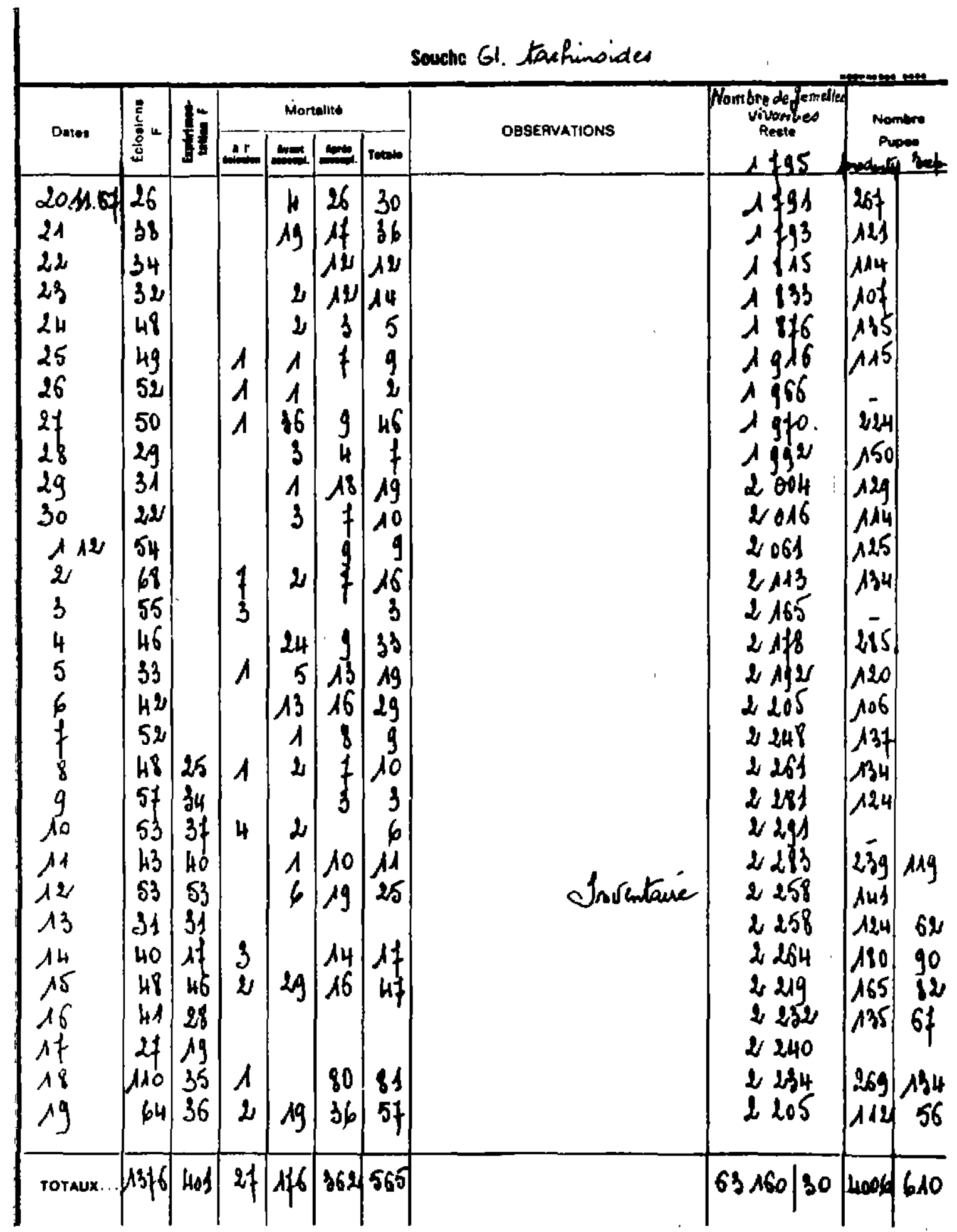

Fig. 3. - Fiche récapitulative pour une période de 30 jours. 
TABLEAU $\mathrm{N}^{\circ} \mathrm{I}$

Elevage de Glossina tachinoïdes

\begin{tabular}{|c|c|c|c|c|c|c|c|c|c|}
\hline \multirow{2}{*}{$\begin{array}{c}\text { Périodes } \\
\text { de } 30 \text { jours } \\
\text { Dates }\end{array}$} & \multirow{2}{*}{$\begin{array}{c}\text { Nombre moyen } \\
\text { de of } \\
\text { vivantes } \\
\text { par jour }\end{array}$} & \multirow{2}{*}{$\begin{array}{c}\text { Pupes } \\
\text { produites } \\
\text { en } 30 \mathrm{j} .\end{array}$} & \multicolumn{3}{|c|}{ Eclosions pupes } & \multirow{2}{*}{$\begin{array}{c}\text { Production } \\
\text { Pupes par } \\
\text { femelle } \\
\text { en } 30 \%\end{array}$} & \multirow{2}{*}{$\begin{array}{c}\text { Total } \\
\text { mortalitê } \\
\text { des } 99\end{array}$} & \multirow{2}{*}{$\begin{array}{c}\text { Mortalité moyenne } \\
\text { journaliêre } \\
\text { Pourcentage du } \\
\text { nombre moyer de } \\
\text { o par Jour } \\
\end{array}$} & \multirow[b]{2}{*}{ observations } \\
\hline & & & $p \cdot 100$ & $\begin{array}{c}\text { Nonbre } \\
0 \\
0\end{array}$ & $\left|\begin{array}{c}\text { Nombre } \\
f\end{array}\right|$ & & & & \\
\hline $\begin{array}{l}26.10 .66 \\
24.11 .66\end{array}$ & 45,1 & 79 & 92,4 & 36 & 37 & 1,7 & 18 & 1,3 & $\begin{array}{l}3.11 .66 \text { nourriture sur lapin } \\
150 \text { pupes reçues le } 5.11 .66\end{array}$ \\
\hline $\begin{array}{l}25.11 .66 \\
24.12 .66\end{array}$ & 94,8 & 177 & 92 & 77 & 86 & 1,8 & 23 & 0,8 & 3.162 pupes reçues $1 \mathrm{e} 15.12 .66$ \\
\hline $\begin{array}{l}25.12,66 \\
23.1 .67\end{array}$ & 530,3 & 683 & 88,7 & 343 & 263 & 1,2 & $488()$. & 3,06 & $\begin{array}{l}\text { H.R. (petite salle) }<80 \text { p. } 100 \text { à } \\
\text { compter du } 16 \text { janvier. }\end{array}$ \\
\hline $\begin{array}{l}24 \cdot 1.67 \\
22.2 .67\end{array}$ & 531,2 & 1.273 & 91,5 & 614 & 551 & 2,3 & 289 & 1,8 & $\begin{array}{l}\text { H.R. (petite salle) }<75 \text { p.100 du } \\
6 \text { au } 18 \text { février }\end{array}$ \\
\hline $\begin{array}{l}23 \cdot 2.67 \\
24.3 .67\end{array}$ & 543,2 & 1.133 & 89,4 & 511 & 502 & 2,08 & 577 & 3,5 & $\begin{array}{l}\text { H.R, (petite salle) }<80 \text { p. } 100 \\
\text { du } 20 \text { févrler au } 13 \text { mars }\end{array}$ \\
\hline 25. 3.67 & 502,1 & 874 & 89,9 & 448 & 338 & 1,74 & 503 & 3,3 & $\begin{array}{l}\text { H,R. (grande salle) }<80 \mathrm{p} \cdot 100 \\
\text { jusqu'au } 17 \text { avr11. }\end{array}$ \\
\hline $\begin{array}{l}24.4 .67 \\
23.5 .67\end{array}$ & 506,1 & 995 & 88,6 & 494 & 388 & 1,96 & 314 & 2,05 & \\
\hline $\begin{array}{l}24 \cdot 5.67 \\
22.6 .67\end{array}$ & 405,9 & 725 & 89,51 & 352 & 297 & 1,78 & 579 & 4,75 & $\begin{array}{l}\text { Intoxication par insecticide du } \\
6.6 .67 \text { au } 17.6 .67\end{array}$ \\
\hline $\begin{array}{l}23.6 .67 \\
22.7 .67\end{array}$ & 373,20 & 799 & 93,61 & 419 & 329 & 2,14 & 212 & 1,89 & $\begin{array}{l}\text { H.R. (petite salle) }>85 \text { p.100 à } \\
\text { compter du } 19 \text { juin. }\end{array}$ \\
\hline $\begin{array}{l}23 \cdot 7.67 \\
21,8.67\end{array}$ & 500,7 & 1.133 & 94,35 & 564 & 505 & 2,26 & 123 & 0,81 & \\
\hline $\begin{array}{l}22.8 .67 \\
20.9 .67\end{array}$ & 754,16 & 1.680 & 93,40 & 784 & 731 & 2,22 & 240 & 1,06 & 58 pupes retirées pour expêrimentation \\
\hline $\begin{array}{l}21.9 .67 \\
20.10 .67\end{array}$ & $1.077,96$ & 2.398 & 93,61 & 1200 & 1045 & 2,22 & 371 & 1,14 & I5 q retirêes pour expérimentation \\
\hline $\begin{array}{l}21.10 .67 \\
19.11 .67\end{array}$ & $1.534,10$ & 2.959 & 93,17 & 1418 & 1339 & 1,92 & 546 & 1,18 & \\
\hline $\begin{array}{l}20.11 .67 \\
19.12 .67\end{array}$ & $2.105,33$ & 4.006 & 91,28 & 1569 & 1531 & 1,90 & 565 & 0,89 & $\begin{array}{l}401 \text { of et } 610 \text { pupes retirées pour } \\
\text { expérimentation }\end{array}$ \\
\hline $\begin{array}{l}20 \cdot 12.67 \\
18.1 .68\end{array}$ & $2.326,20$ & 4.427 & 92,30 & 897 & 782 & 1,90 & 974 & 1,39 & $\begin{array}{l}259 \text { ot } 2.608 \text { pupes retirées pour } \\
\text { expéximentation. }\end{array}$ \\
\hline
\end{tabular}

(.) dont 364 femelles morteg avant accouplement. 


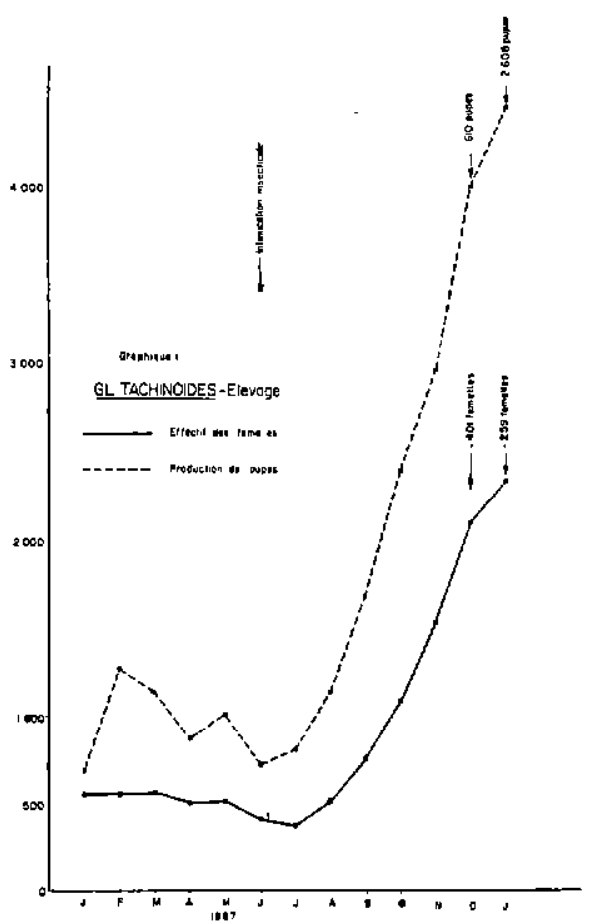

Dans le tableau 1, où sont mentionnés les résultats obtenus entre le 26 octobre 1966 et le 18 Janvier 1968, les observations sont rassemblées par pérıode de 30 jours. Nous y indiquons le nombre moyen de femelles vivantes par jour, pour chaque période de 30 jours : la production totale de pupes pendant la même période, le pourcentage d'éclosion ef le nombre de males et de femelles issus de ces pupes ; la production de pupes par femelle pour 30 jours : le total des femelles mortes au cours du mois correspondant ef le pourcentage moyen quotidien de mortalité.

On constate qu'à partir de la fin de l'année 1966 les effectifs des femelles augmentent brusquement et passent de moins de 100 individus à plus de 500 femelles en un mois.

Cet accroissement des effectifs est dô aux éclosions massives des femeiles issues des pupes reçues en décembre 1966.

Puis, pendant quatre mois, les effectifs restent stables ou subissent une légère baisse. A cette époque divers incıdents, qui sont détaillés dans le paragraphe suivant, ont provoqué une augmentation de la mortalité.

Les effectifs tombent ensuite, en deux mois, à moins de 400 femelles/jour. C'est à cette époque que se situe une intoxication accidentelle par insecticide dont les effets seront exposés dans la suite de cet article.

Les pupes produites n'ayant pas été en contact avec l'insecticide, grace au stockage dans ia petite salle, permettent un relèvement des effectifs dès la fin juillet 1967. Ces effectifs s'accroissent alors de façon régulière et l'on peut avancer que si nous avions conservé toutes les femelles écloses et toutes les pupes produites, nous aurions atteint 10.000 femelles/jour au cours du mois d'avril 1968, soit en moins de 10 mois.

La production de pupes suit une progression parallèle à celle des effectifs et dépasse 4.000 pupes par mois à la fin de l'année 1967. Dans la même hypothèse que ci-dessus, on peut estimer que la production mensuelle aurait approché 20.000 pupes en avril 1968. La lecture du tableau I montre en effet que le nombre de pupes par femelle vivante en 30 jours oscille autour de 2 à partır de la fin juin 1967.

\section{B. - Longévité des femelles et production de pupes.}

Nous avons figuré, dans le graphique 2 , la durée de survie 50 p. $100 *$ des femelles accouplées, d'une part, et des femelles âgées de plus de 10 jours donc en âge de reproduire, d'autre part, pour chacun des lots de femelles écloses entre la fin décembre 1966 et la fin octobre 1967.

Dans le graphique 3, nous avons reporté le nombre moyen de pupes par femelle, en 150 jours, pour chacun des lots correspondants.

Le nombre moyen de pupes par femelle a été calculé en tenant compte de la mortalité des femelles à partir du 11 e jour qui suit la date d'éclosion. Pour ce faire, nous avons totalisé le nombre de femelles vivantes chaque jour, entre le jour 11 et le jour 150, ou entre le jour 11 ef le jour aù la dernière femelle du lot est morte, si les femelles de ce lot ont vécu moins de 150 jours. Le chiffre obłenu, divisé par 140, ou par le nombre de jours compris entre le jour 11 et le dernier jour du lot, est rapporté au nombre total de pupes produites pendant la période consi-

* Nous entendons par durée de survie 50 p. 100 le temps au bout duquel il reste la moitié du nombre initial d'individus vivants. 


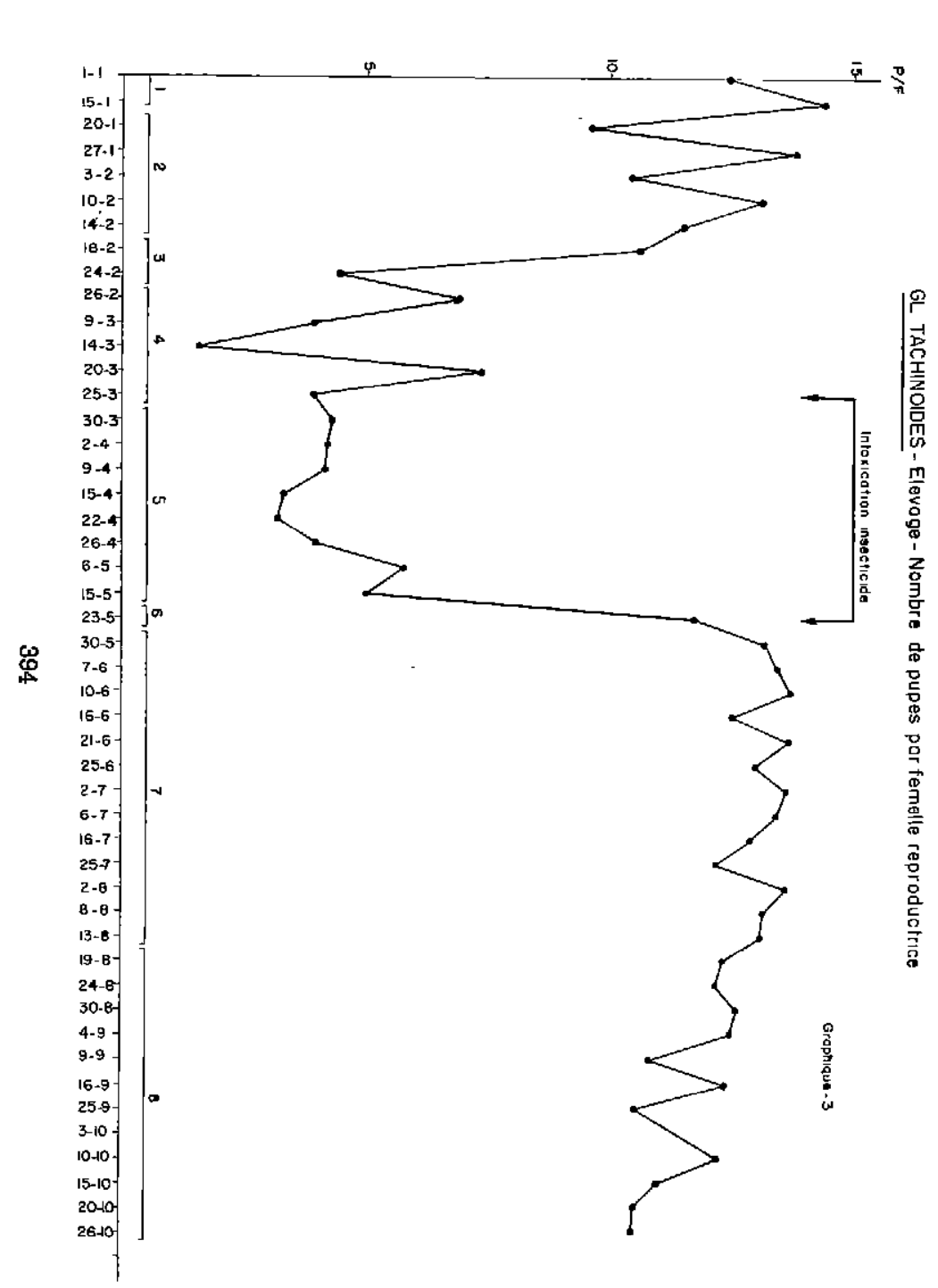

号命

प्.
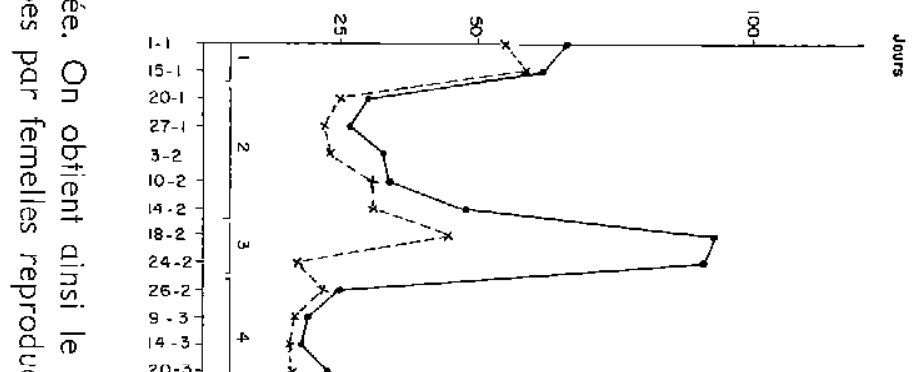

产.

은

0.3
-00

$\pi \stackrel{\mathscr{T}}{3}$

무음

品

勇

竞

号

음

芩号

옹

क祭

$16-9$

邑是 25-9

总亮

$3-10$
$10-10$
15.10
20.10

当 N

동 
TABLEAU N*II

\begin{tabular}{|c|c|c|c|}
\hline Përiode & $\begin{array}{l}\text { Dates d'écloston } \\
\text { des femelles }\end{array}$ & $\begin{array}{l}\text { Nombre de } \\
\text { femelles }\end{array}$ & Observations \\
\hline 1 & avant le 17.1 .67 & 670 & $\begin{array}{l}\text { Femelles issues des lots de pupes du } 5 \text { novembre } \\
\text { et } 15 \text { décembre } 1966 \text {. }\end{array}$ \\
\hline 2 & du 17.1 au 17.2 .67 & 192 & H.R. < 75 p.100 (Humidiflcateur dêfectueux) \\
\hline 3 & du 18.2 au 24.2 .67 & 67 & H.R. < 80 p. 100 (Humidistat mal rëglê) \\
\hline 4 & $\mathrm{du} 26.2$ au 27.3 .67 & 326 & début probable de 1 'intoxication par insecticlde \\
\hline 5 & du 28.3 au 18.5 .67 & 486 & Intoxication par insecticlde \\
\hline 6 & du 19.5 au 27.5 .67 & 54 & Fin intoxication par insecticide \\
\hline 7 & du 28.5 au 18.8 .67 & 769 & \\
\hline B & du 19.8 au 30.10 .67 & 1459 & $\begin{array}{l}\text { Pas d'alimentation le dimanche. } \\
\text { Femelles de plus de } 170 \text { jours sacrifiêes. }\end{array}$ \\
\hline
\end{tabular}

périodes caractérisées chacune par la survie 50 p. 100 et per le P. F. R. (tableau II).

La période 1 comprend toutes les femelles issues des lots de pupes reçues en novembre et décembre 1966. Elle se caractérise par une survie 50 p. 100 comprise entre 55 et 65 jours et un $P$. F. R. compris entre 12,4 et 14,4 .

La période 2 se caractérıse par une survie $50 \mathrm{p.} 100$ n'atteignent pas 50 jours et le plus souvent inférieure à 35 jours, et un $P . F . R$. compris entre 9 et 13,8 . Pendant cette période, par suite du mauvais fonctionnement de l'un des humidificateurs, l'humidité relative des deux sailes d'élevage fut inférieure à 75 p. 100.

La période 3 est caractérisée par une forte mortalıté des femelles de moins de 10 jours (survie 50 p. 100 des femelles accouplées comprise entre 17 et 45 jours), et une bonne survie des femelles en âge de reproduire (survie 50 p. 100 supérieure à 90 jours). Le P. F. R. passe, au cours de la même période, de 10,6 à 4,4. A cette époque I'humidificateur avait été réparé, mass par suite d'un mauvais réglage de l'humıdistat, I'H. $R$. de la petite salle est restée inférieure à 80 p. 100.

Les femelles correspondant à la période 4 ont une longévité très faible (survie 50 p. 100 comprise entre 16 et 31 jours) et le P. F. R. oscille entre 1,5 et 7,3 . II est probable qu'au cours de cette période l'intoxication par insecticide avaił déjà commencé de façon insıdieuse. et que ses effets se sont ajoutés à ceux provoqués par une H. R. insuffisante.

Les femelles de la période 5 ont été intoxiquées de façon certaıne par des émanations d'insec- ticide. La longévité est rédulte (longévité maximum ne dépassant pas 80 jours ; survie 50 p. 100 comprise entre 15 et 57 jours). Le P. F. R. est faible, et voisin de 4.

Les femelles de la période 6 n'ont été intoxiquées par l'insecticide qu'au début de leur vie, entre 10 et 20 jours. La longévité augmente (longévité maximum atteignant 150 jours : survie 50 p. 100 égale à 30 jours). Le P. R. F. atteint 11,7 .

Pendant la période 7 , les femelles ont une très bonne longévité (survie 50 p. 100 entre 75 et 110 jours) et le P. F. R. oscille autour de 13. A cette époque, grâce à l'achat d'un nouvel humidificateur, le 19 juin, I'H. R. de la petite salle reste supérieure à 85 p. 100.

Au cours de la période 8 la longévité reste bonne mais le $P$. F. R. diminue légèrement et passe de 12,5 à 10,5. C'est au cours de cette période, alors que les femelles avaient entre 130 et 60 jours, que les mouches n'ont plus été nourries le dimanche. En outre les femelles âgées de plus de 170 jours ont été sacrifiées.

La longévité ef la production de pupes ramenées à 100 femelles, pour chacune des 8 périodes ainsi définies, sont rassemblées dans le tableau III et les graphiques 4 et 5 .

On constate que les périodes 1,7 et 8 sont assez semblables, la mellleure langévité ef la meilleure production de pupes ayant été abtenues au cours de la période 7.

C'est au cours de la période 4 que les résultats sont les plus médiocres.

Au cours de la période 5, époque où les femelles ont pleinement subı l'intoxication par insecticide, 
TABLEAU $\mathrm{N}^{\circ}$ III

Glossina tachinoïdes - Longévité des femelles

Production de pupes

\begin{tabular}{|c|c|c|c|c|c|c|c|c|c|c|c|c|c|c|c|c|}
\hline \multirow{2}{*}{ Jours } & \multicolumn{4}{|c|}{ lère période } & \multicolumn{4}{|c|}{ 2ème période } & \multicolumn{4}{|c|}{ 3ème période } & \multicolumn{4}{|c|}{ 4ème période } \\
\hline & 1 & 2 & 3 & 4 & $I$ & 2 & 3 & 4 & 1 & 2 & 3 & 4 & 1 & 2 & 3 & 4 \\
\hline 0 & 670 & 100,00 & & & 192 & 100,00 & & & 67 & 100,00 & & & 326 & 100,00 & & \\
\hline $1-10$ & 576 & 85,97 & & & 165 & 85,93 & & & 57 & 85,07 & & & 283 & 86,80 & & \\
\hline $11-20$ & 551,6 & 82,32 & 361 & 53,83 & 146,5 & 76,30 & 87 & 45,24 & 47,0 & 70,14 & Il & 16,41 & 205,0 & 62,88 & 22 & 6,72 \\
\hline $21-30$ & 494,7 & 73,83 & 447 & 120,49 & 104,9 & 54,63 & 102 & 98,34 & 31,9 & 47,61 & 14 & 37,26 & 88,9 & 27,26 & 33 & 16,83 \\
\hline $31-40$ & 463,9 & 69,23 & 468 & 190,27 & 81,6 & 42,50 & 90 & 145,17 & 27,5 & 41,04 & 25 & 74,56 & 66,4 & 20,36 & 41 & 29,39 \\
\hline $41-50$ & 399,1 & 59,56 & 400 & 249,95 & 72,4 & 37,70 & 73 & 183,17 & 25,5 & 38,05 & 23 & 108,84 & 63,9 & 19,60 & 40 & 41,64 \\
\hline $51-60$ & 340,1 & 50,76 & 321 & 297,81 & 67,0 & 34,89 & 75 & 222,21 & 24,9 & 37,16 & 29 & 152,09 & 60,2 & 18,46 & 51 & 57,27 \\
\hline $61-70$ & 283,3 & 42,28 & 275 & 338,82 & 61,3 & 31,92 & 65 & 256,04 & 24,0 & 35,82 & 20 & 181,92 & 54,7 & 16,77 & 38 & 68,90 \\
\hline $71-80$ & 240,1 & 35,83 & 258 & 377,30 & 60,4 & 31,45 & 56 & 285,19 & 24,0 & 35,82 & 29 & 225,19 & 43,2 & 13,25 & 41 & 81,47 \\
\hline $81-90$ & 216,6 & 32,32 & 221 & 410,20 & 54,7 & 28,48 & 63 & 317,97 & 23,0 & 34,32 & 18 & 252,02 & 29,8 & 9,14 & 24 & 88,82 \\
\hline $91-100$ & 183,7 & 27,41 & 170 & 435,61 & 51,9 & 27,03 & 56 & 347,10 & 16,7 & 24,92 & 22 & 284,84 & 16,4 & 5,03 & 12 & 92,49 \\
\hline $101-110$ & 161,5 & 24,10 & 152 & 458,28 & 41,6 & 21,66 & 42 & 368,95 & 10,7 & 15,97 & 9 & 298,27 & 4,2 & 1,28 & 3 & 93,40 \\
\hline $111-120$ & 140,3 & 20,94 & 123 & 476,62 & 26,0 & 13,54 & 25 & 381,96 & 2,6 & 3,88 & & & 1,0 & 0,30 & & \\
\hline $121-130$ & 118,3 & 17,65 & 97 & 491,07 & 11,6 & 6,04 & 7 & 385,60 & & & & & & & & \\
\hline $131-140$ & 106,6 & 15,91 & 72 & 501,81 & 3,8 & 1,97 & 2 & 386,63 & & & & & & & & \\
\hline $141-150$ & 85,2 & 12,71 & 43 & 508,21 & 1,0 & 0,52 & & & & & & & & & & \\
\hline $151-160$ & 55,0 & 8,20 & 18 & 510,89 & & & & & & & & & & & & \\
\hline $161-170$ & 8,4 & 1,25 & 4 & 511,48 & & & & & & & - & & & & & \\
\hline $\begin{array}{l}171-180 \\
181-190\end{array}$ & 1,0 & 0,14 & 1 & 511,62 & & & & & & & & & & & & \\
\hline $191-200$ & & & & & & & & & & & & & & & & \\
\hline
\end{tabular}


TABLEAU N ${ }^{\star}$ III (suite)

\begin{tabular}{|c|c|c|c|c|c|c|c|c|c|c|c|c|c|c|c|c|}
\hline \multirow[b]{2}{*}{ Jours } & \multicolumn{4}{|c|}{ Sēme période } & \multicolumn{4}{|c|}{ 6ème përiode } & \multicolumn{4}{|c|}{ 7ème période } & \multicolumn{4}{|c|}{ 8ème pêriode } \\
\hline & 1 & 2 & 3 & 4 & 1 & 2 & 3 & 4 & 1. & 2 & 3 & 4 & I & 2 & 3 & 4 \\
\hline $\begin{array}{r}1-10 \\
11-20\end{array}$ & $\begin{array}{l}412 \\
342,1\end{array}$ & $\begin{array}{l}84,77 \\
70,39\end{array}$ & & & 49 & $\begin{array}{l}90,74 \\
84,81\end{array}$ & & & 682 & 88,68 & & & 1283 & 87,93 & & \\
\hline $\begin{array}{l}11-20 \\
21-30\end{array}$ & 260,9 & $\begin{array}{l}70,39 \\
53,68\end{array}$ & $\begin{array}{l}118 \\
213\end{array}$ & $\begin{array}{l}24,21 \\
68,01\end{array}$ & 45,8 & $\begin{array}{l}84,81 \\
59,25\end{array}$ & $\begin{array}{l}39 \\
25\end{array}$ & 72,17 & 651,3 & 84,69 & 470 & 61,06 & 1261,3 & 86,44 & 839 & 57,48 \\
\hline $\begin{array}{l}21-30 \\
31-40\end{array}$ & 213,2 & & $\begin{array}{l}213 \\
170\end{array}$ & $\begin{array}{r}68,01 \\
102,96\end{array}$ & 32,0 & $\begin{array}{l}59,25 \\
40,00\end{array}$ & 25 & 118,44 & 609,6 & 79,27 & 584 & 137,00 & 1209,9 & 82,92 & 1050 & 129,37 \\
\hline $41-50$ & 149,2 & 30,69 & 116 & $\begin{array}{l}102,96 \\
126,80\end{array}$ & 21,6 & $\begin{array}{l}40,00 \\
29,25\end{array}$ & $\begin{array}{l}23 \\
18\end{array}$ & 161,00 & 568,9 & 73,97 & 583 & 212,74 & 1141,0 & 78,20 & 1038 & 200,45 \\
\hline $51-60$ & 103,6 & 21,31 & 89 & $\begin{array}{l}126,80 \\
145,10\end{array}$ & 15,8 & 29,25 & 18 & 194,31 & 538,5 & 70,02 & 544 & 283,46 & 1075,4 & 73,70 & 901 & 262,13 \\
\hline $61-70$ & 40,7 & 8,37 & 25 & $\begin{array}{l}145,10 \\
150,24\end{array}$ & 10,8 & 20,00 & 9 & 210,97 & 519,8 & 67,59 & 505 & 349,09 & 998,9 & 68,46 & 812 & 317,72 \\
\hline $71-80$ & 13,0 & 2,67 & 5 & & $\begin{array}{r}10,0 \\
9,2\end{array}$ & 38,51 & 7 & 223.92 & 488,7 & 63,55 & 509 & 415,24 & 917,8 & 62,90 & 808 & 373,07 \\
\hline $81-90$ & & & & & $\begin{array}{l}9,2 \\
8,1\end{array}$ & 17,03 & $\begin{array}{r}11 \\
4\end{array}$ & 244,27 & 442,7 & 57,56 & 443 & 472,80 & 822,3 & 56,36 & 734 & 423,34 \\
\hline $91-100$ & & & & & $\begin{array}{l}8,1 \\
7,0\end{array}$ & $\begin{array}{l}15,00 \\
12,96\end{array}$ & $\begin{array}{l}4 \\
5\end{array}$ & 251,66 & 397,5 & 51,69 & 390 & 523,50 & 724,1 & 49,62 & 645 & 467,50 \\
\hline $101-110$ & & & & & $\begin{array}{l}7,0 \\
7,0\end{array}$ & $\begin{array}{l}12,96 \\
12,96\end{array}$ & $\begin{array}{l}5 \\
7\end{array}$ & 260,91 & 349,7 & 45,47 & 356 & 569,78 & 635,0 & 43,52 & 576 & 506,97 \\
\hline $111-120$ & & & & & $\begin{array}{l}7,0 \\
6,0\end{array}$ & 12,96 & $\begin{array}{l}7 \\
2\end{array}$ & 273,87 & 297,7 & 38,71 & 280 & 606,16 & 524,4 & 35,94 & 466 & 538,88 \\
\hline $121-130$ & & & & & $\begin{array}{l}6,0 \\
5,0\end{array}$ & $\begin{array}{r}11,11 \\
9,25\end{array}$ & $\begin{array}{l}2 \\
3\end{array}$ & 277,57 & 246,6 & 32,06 & 236 & 636,84 & 427,7 & 29,31 & 346 & 562,56 \\
\hline $131-140$ & & & & & $\begin{array}{l}5,0 \\
5,0\end{array}$ & 9,25 & $\begin{array}{l}3 \\
4\end{array}$ & 283,12 & 202,6 & 26,34 & 179 & 660,09 & 352,6 & 24,16 & 264 & 580,63 \\
\hline $141-150$ & & & & & $\begin{array}{l}5,0 \\
4,3\end{array}$ & $\begin{array}{l}9,25 \\
7,96\end{array}$ & $\begin{array}{l}4 \\
1\end{array}$ & 290,52 & 151,8 & 19,73 & 126 & 676,46 & 273,3 & 18,73 & 190 & 593,64 \\
\hline $151-160$ & & & & & $\begin{array}{l}4,3 \\
4,0\end{array}$ & $\begin{array}{l}7,96 \\
7,40\end{array}$ & $\begin{array}{l}1 \\
1\end{array}$ & 292,36 & 106,1 & 13,79 & 71 & 685,68 & 176,2 & 12,07 & 85 & 599,45 \\
\hline $161-170$ & & & & & $\begin{array}{l}4,0 \\
3,1\end{array}$ & $\begin{array}{l}7,40 \\
5,74\end{array}$ & $\begin{array}{l}1 \\
3\end{array}$ & 294,21 & 65,2 & 8,47 & 35 & 690,21 & 62,7 & $\begin{array}{l}4,29 \\
0,86\end{array}$ & $\begin{array}{r}35 \\
7\end{array}$ & 601,84 \\
\hline $171-180$ & & & & & $\begin{array}{l}3,1 \\
1,3\end{array}$ & $\begin{array}{l}5,74 \\
2,40\end{array}$ & $\begin{array}{l}3 \\
1\end{array}$ & 299,76 & 33,6 & 4,36 & 18 & 692,54 & 12,6 & 0,86 & 7 & 602,31 \\
\hline $181-190$ & & & & & $\begin{array}{l}1,3 \\
1,0\end{array}$ & $\begin{array}{l}2,40 \\
1,85\end{array}$ & $\begin{array}{l}1 \\
1\end{array}$ & 301,60 & 22,9 & 2,97 & 17 & 694,74 & & & & \\
\hline $191-200$ & & & & & $\begin{array}{l}1,0 \\
1,0\end{array}$ & $\begin{array}{l}1,85 \\
1,85\end{array}$ & 1 & 303,45 & $\begin{array}{r}10,3 \\
3,0\end{array}$ & $\begin{array}{l}1,33 \\
0,39\end{array}$ & $\begin{array}{l}9 \\
1\end{array}$ & $\begin{array}{l}695,63 \\
695,76\end{array}$ & & & & \\
\hline $201-210$ & & & & & & 1,00 & & & 1,0 & & & & & & & \\
\hline
\end{tabular}

1 = Nombre moyen de femelles/jour par décade. 2 = Pourcentage de femelles vivantes. 3 = Nombre de pupes produites par décade,

4 - Nombre de pupes pour 100 femelles (totaux cumulés). 


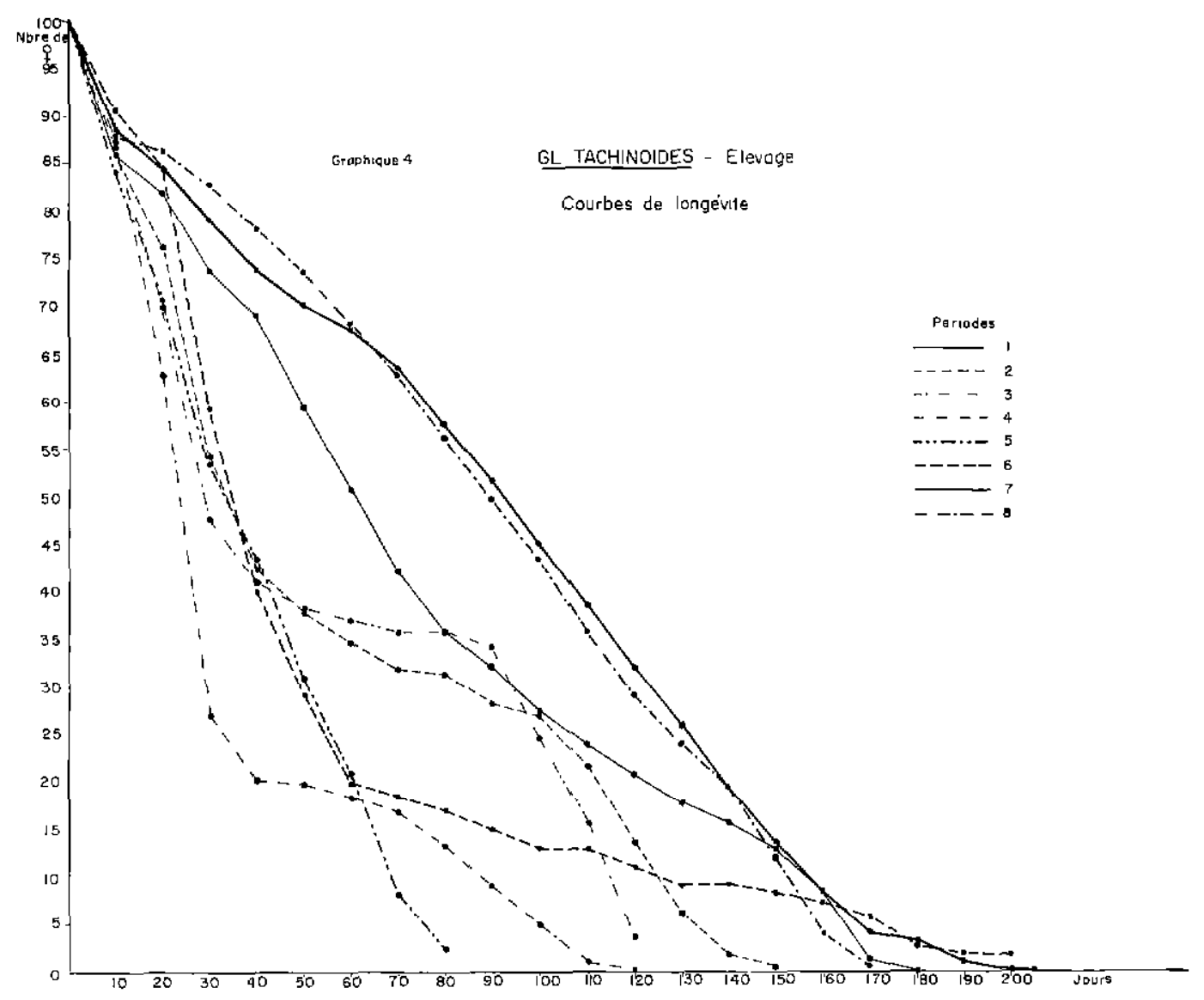

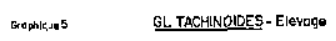

Production de pupes pour 100 i

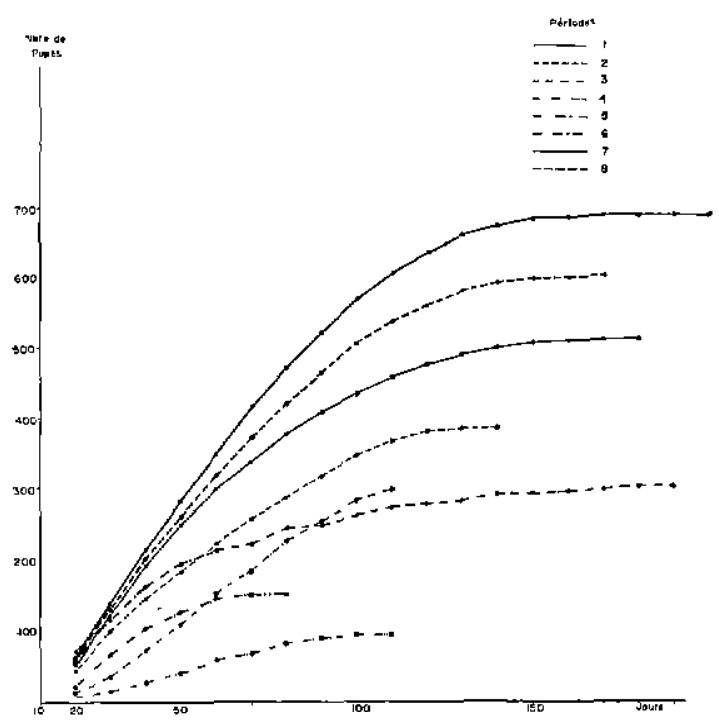

les résultats sont un peu meilleurs que pendant la période 4, mais moins bons que pendant les périodes 2, 3 et 6 .

$\mathrm{Si}$ on examine les résultats obtenus au cours de la période 7, qui débute fin mai 1967 et se termine le 10 Janvier 1968, avec la mort des dernières femelles, on constate que, pour l'ensemble des femelles de cette période, au 60 e jour survant l'éclosion, 67,6 p. 100 des femelles sont vivantes et que la production de pupes. pour 100 femelles accouplées, atteint 349 pupes. 50 p. 100 des femelles sont encore en vie au 90 e jour et la longévité maximum atteint 210 jours. La production totale de pupes a atteint, à cette date, 5.356 pupes pour 769 femelles accouplées, soit 695 pupes pour 100 femelles.

Ces chiffres sont inférieurs à ceux obtenus par JORDAN, NASH et BOYLE (1967) avec Gl. austeni nourrie sur lapin (91 p. 100 de femelles 
vivantes au 60 jour ; 402 pupes produites au 58 jour, pour 100 femelles; longévité maximum : 278 jours ; production totale de pupes, pour 100 femelles : 1.142), ce que nous attribuons d̀ une moins bonne survie de nos femelles au cours des premières décades. La courbe de longévité décroît en effet régulièrement (graphique 4), alors que, chez Gl. austeni, la mortalité est très faible jusque vers le $70^{\mathrm{e}}$ jour.

Néanmoins les résultats obtenus avec les femelies de cette $7 \mathrm{e}$ période sont nettement meilleurs que ceux obtenus par JORDAN, NASH ef BOYLE avec Gl, austeni nourrie sur chèvre (57 p. 100 de survie du 60 jour ; 272 pupes pour 100 femelles au $58^{\mathrm{e}}$ jour ; longévité maximum ; 176 jours : production totale de pupes, pour 100 femelles : 536).

La 8e période débute le 19 août 1967 et se termine le 15 février 1968. Au 60 e jour sulvant l'éclosion 68,4 p. 100 des femelles sont vivantes et la production de pupes atteint 317 pupes pour 100 femelles accouplées, 50 p. 100 des femelles sont en vie au 80 e jour. La production totale de pupes atteint, au 170e jour, 8.796 pupes pour 1.459 femelles accouplées, soit 602 pupes pour 100 femelles.

\section{C. - Poids des pupes.}

Des pupes, âgées au maximum de 24 heures, ont été pesées à quatre époques différentes (tableau IV).

La première pesée, effectuée en décembre 1966, concerne les pupes produites par les femelles antérieures à l'envoi de pupes du 15 décembre 1966. Ces femelles étaient déjà nourries sur oreilles de lapin.

La deuxième pesée, effectuée en Janvier 1967. concerne les pupes produites par les femelles de la période 1 .
La troisième pesée, effectuée en mars 1967. concerne les pupes produites par les femelles de lo période 4.

La quatrième pesée, effectuée en juın 1967. concerne les pupes produites par les femelles. des périodes 5 et 6 .

L'ensemble de ces pesées donne un poids moyen oscillant autour de $16,2 \mathrm{mg}$ (écart type de la moyenne : 0,08602), avec un minimum de $8 \mathrm{mg}$ et un maximum de $24 \mathrm{mg}$.

BUXTON et LEWIS (1934) trouvent un poids moyen, pour des pupes de 24 heures de $G$. tochinoides élevées à $24^{\circ} \mathrm{C}$, de $15,11 \mathrm{mg}$ aver des variations extrêmes de 6 à $23 \mathrm{mg}$ (erreur standard $\pm 0,086$ ).

\section{IV. - INTOXICATION PAR INSECTICIDE}

Les 5 ef 6 Juin 1967 nous sommes alertés par une mortalıté un peu plus élevée que'les jours. précédents, mais, à partir du 7 juin, cette mortalité prend des proportions très élevées qui dépassent 10 p. 100 de l'effectif des femelles reproductrices. Une enquête entreprise aussitôt nous fait découvrir que, à notre insu, on avait disposé dans différents bureaux avoisinants notre salie d'élevage des plaquettes insecticides Vapona (marque déposée Shell) à base de diméthyl dichlorovinyl phosphate, pour détruire les mouches domestıques abondantes à cette époque de l'année.

II est probable que l'insecticide a été véhiculé des bureaux à la salle d'élevage par le personnel chargé du nettoyage ou par des courants d'air à la faveur de l'ouverture des pories.

Les plaquettes Vapona ont été immédiatement retirées, mais la mortalité est restée supérieure à la normale jusqu'au 16 juin et $c_{\text {, par la suite }}$ dépassé, de temps à autres, 2 p. 100 de l'effectıf

TABLEAU $N^{\circ} I V$

\begin{tabular}{|c|c|c|c|c|}
\hline Date de pesée & $\begin{array}{c}\text { Nombre de } \\
\text { pupes }\end{array}$ & $\begin{array}{c}\text { Poids moyen } \\
\text { (en mg) }\end{array}$ & $\begin{array}{c}\text { Ecarr-type de } \\
\text { l'échantillon }\end{array}$ & $\begin{array}{c}\text { Ecart-type de } \\
\text { la moyenne }\end{array}$ \\
\hline Décembre 1966 & 180 & 16,70 & 2,114 & 0,15748 \\
Janvier 1967 & 103 & 16,09 & 1,816 & 0,17888 \\
Mars 1967 & 116 & 16,06 & 2,742 & 0,25455 \\
Juin 1967 & 243 & 15,95 & 2,032 & 0,13038 \\
\hline Total des pesêes & 642 & 16,20 & 2,186 & 0,08602 \\
\hline
\end{tabular}


TABLEAU $\mathrm{N} \bullet \mathrm{V}$

Mortalité des femelles reproductrices de Glossina tachinotdes au cours du mols de juin 1967

\begin{tabular}{|c|c|c|c|}
\hline Dates & $\begin{array}{l}\text { Nombre de femelles } \\
\text { reproductrices }\end{array}$ & $\begin{array}{c}\text { Nombre de femelles } \\
\text { mortes }\end{array}$ & $\begin{array}{c}\text { Pourcentage de mortalité } \\
\text { quotidienne }\end{array}$ \\
\hline $\begin{array}{l}1.6 .67 \\
2.6 .67 \\
3.6 .67 \\
4.6 .67 \\
5.6 .67 \\
6.6 .67 \\
7.6 .67 \\
8.6 .67 \\
9.6 .67 \\
10.6 .67 \\
11.6 .67 \\
12.6 .67 \\
13.6 .67 \\
14.6 .67 \\
15.6 .67 \\
16.6 .67 \\
17.6 .67 \\
18.6 .67 \\
19.6 .67 \\
20.6 .67 \\
21.6 .67 \\
22.6 .67 \\
23.6 .67 \\
24.6 .67 \\
25.6 .67 \\
26.6 .67 \\
27.6 .67 \\
28.6 .67 \\
29.6 .67 \\
30.6 .67\end{array}$ & $\begin{aligned} & 446 \\
& 440 \\
& 437 \\
& 433 \\
& 424 \\
& 414 \\
& 400+ 61=461 \quad(.) \\
& 411 \\
& 384 \\
& 335 \\
& 309 \\
& 275 \\
& 241+ 55=296(.) \\
& 229 \\
& 206 \\
& 188 \\
& 175 \\
& 175 \\
& 175 \\
& 173+ 50=223(.) \\
& 216 \\
& 208 \\
& 205+ 54=259(.) \\
& 256 \\
& 255 \\
& 248 \\
& 241 \\
& 234 \\
& 231+ 52=283(.) \\
& 281\end{aligned}$ & $\begin{array}{r}6 \\
3 \\
4 \\
9 \\
10 \\
14 \\
50 \\
27 \\
49 \\
26 \\
34 \\
34 \\
67 \\
23 \\
18 \\
13 \\
0 \\
0 \\
2 \\
7 \\
8 \\
3 \\
3 \\
1 \\
7 \\
7 \\
7 \\
3 \\
2 \\
2\end{array}$ & $\begin{array}{r}1,34 \\
0,68 \\
0,91 \\
2,07 \\
2,35 \\
3,38 \\
10,84 \\
6,56 \\
12,76 \\
7,76 \\
11,00 \\
12,36 \\
22,63 \\
10,04 \\
8,73 \\
6,91 \\
0 \\
0 \\
1,14 \\
3,13 \\
3,70 \\
1,44 \\
1,15 \\
0,39 \\
2,74 \\
2,82 \\
2,90 \\
1,28 \\
0,70 \\
0,71\end{array}$ \\
\hline
\end{tabular}

(.) = Jeunes femelles introduites dans le lot des femelles reproductrices après séparation d'avec les mâles.

des femelles reproductrices, jusqu'au 27 juin (tableau $V$ ).

Seules les femelles reproductrices ont été ulleintes. Les jeunes femelles de mains de 10 jours et les pupes n'ont pas été en contact avec l'insecticide grâce à l'isolement de la petite salle.

Des différentes espèces en élevage, c'est Gl. tochinordes qui a le plus souffert de l'intoxication. Alors que la mortalité moyenne par jour atteint 4,75 p. 100 de l'effectif total des femelles, entre le 24 mai et le 22 juin, chez $G$. tachinordes, elle n'est que de 2,18 p. $100 \mathrm{chez}$ Gl. morsitans souche de Rhodésie, de 2,14 p. 100 chez Gl. morsitans souche du Tanganyika, ef 1,05 p. 100 chez Gl. austeni.

Les femelles reproductrices de ces différentes espèces sont toutes stockées dans la même salie et nourries sur les mêmes animaux. II faut donc en conclure d'une part que les quantités d'insec- ticide introduites dans la salle d'élevage ont été très faibles, puisqu'elles ont très peu affectées Gl. mortisans et Gl. austeni, et d'autre part que Gl. tachinoides est particulièrement sensıble à l'action des insecticides.

Il est très vraisemblable enfin que l'intoxication a commencé bien avant le mois de juin. On remarque en effet que les femelles de la période précédent l'ıntoxication (4e période) ont une longévité réduite et une très faible production de pupes. Les femelles de cette période ont dû être affectées d̀ la fois par le mauvais fonctionnement de l'appareil humidificateur et par de faibles quantités d'insecticides introduites occasionnellement. Ces facteurs ont agi de façon insidieuse ef n'ont jamais provoqué la mortalité brutale qui nous a alerté en juin.

Les femelles affectées par l'intoxication décelée en juin appartiennent aux $5 \mathrm{e}$ et $6 \mathrm{e}$ périodes. Les femelles de la $5 \mathrm{e}$ période ont une longévité 
très réduite, qui ne dépasse pas 80 jours, avec une survie 50 p. 100 qui n'atteint pas 40 jours (tableau III). La production totale de pupes n'est que de 151 pupes pour 100 femelles accouplées. Ces femelles avaient entre 70 et 30 jours au moment de l'accident.

Au cours de la Ge $^{\mathrm{e}}$ période les femelles, qui n'ont été atteintes par l'insecticide qu'au cours de la deuxième décade de leur vie, ont un meilleure longévité (longévité maxımum = 200 jours), bien que la survie 50 p. 100 ne dépasse pas 31 jours. La production totale de pupes, pour 100 femelles, atteint 303 pupes.

A la différence des constatations effectuées par AZEVEDO et PINHAO (1967), qui ont eu un accident analogue avec leur élevage de Lisbonne, l'insecticide ne semble avoir eu qu'une faible influence sur les taux d'éclosion des pupes produites (tableau 1). Si l'on remarque une baisse du pourcentage d'éclosion avant l'accident (88,6 p. 100 d'éclosion entre le 24 avril et le 23 mai 1967), ce taux est de 89,51 p. 100 entre le 24 mai et le 22 juin et dépasse 94 p. 100 au cours du mois de juillet 1967, pour osciller pendant les mois suivants entre 91 et 93 p. 100 . Il est vraisemblable que le fait de récolter les pupes chaque matin et de les transférer aussitôt dans la salle voisine a soustrait ces pupes à l'action néfaste de l'insecticide.

\section{V. - DISCUSSION}

Les résultats obtenus par NASH et ses collaborateurs avec Gl. ousteni démontrent de façon évidente que l'utilisation du lapin comme animalhôte constitue un facteur primordial dans la réussite complète d'un élevage de glossınes. Ces auteurs, à la suite de différents essais comparatifs, estıment en outre que le volume nécessaire à chaque mouche doit être de l'ordre de $64 \mathrm{~cm}^{3}$, et que ces insectes doivent être nourris chaque jour, à l'exception du dimanche, pendant 15 minutes sur le lapin.

La surface de nos installations ne pouvant, dans l'immédiat, être augmentée, et le personnel dont nous disposons ne pouvant être accru, nous avons dû limiter le volume accordé à chaque mouche à environ $30 \mathrm{~cm}^{3}$ et ne laisser les mouches sur le lapin que pendant 4 minutes au maximum.
Nous avons donc quelque peu sacrifié le rendement au bénéfice de la rapidité et de la simplification des méthodes.

L'objectıf essentiel de NASH est d'atieindre le potentiel reproducteur maximum de l'espèce qu'il élève.

Les élevages que nous avons entrepris à Maisons-Alfort ne peuvent viser exactement au même but. Sı nous cherchons à obtenir une longévité maxımum ef une production de pupes élevées, notre principal abjectif est de récliser, dans des conditions pouvant être facilement utilisées dans la pratique, des élevages à effectifs nombreux, fournissant un matériel abondant en vue d'effectuer diverses expérimentations.

Les résultats que nous avons obtenus, sans être aussi excellents que ceux de BRISTOL, sont cependant très encourageants.

La réussite de l'élevage de Gl. tachinordes est incontestablement due à l'adoption du lapin comme anımal-hôte. Ce facteur, pour primordial qu'il soit, n'est cependant pas le seul à avoir eu une influence bénéfique. Le taux d'humidité joue également, chez $\mathrm{Gl}$. tochinoides, un rôle très important. Les résultats médiocres obtenus pendant les périodes 2 et 3 , alors que l' $H$. R. est restée inférieure d̀ 80 p. 100, montrent que $G$ l. tachinoides a besoin, plus que Gl. morsitans, d'une humidité élevée, ce qui est du reste tout à fait en accord avec les observations écologiques effectuées, dans la nature, chez cette espèce.

Gl. tochinoides paraît en outre être une espèce fragile, très sensible aux facteurs défavorables. Elle semble en particulier beaucoup plus sensible que Gl. morsitons à l'action des insecticides, tout au moins dans les conditions que nous avons observées.

\section{VI. - CONCLUSIONS}

Après avoir, pendant 21 mois, maintenu difficilement un élevage de Gl. tachinoides nourries successivement sur cobaye ef sur poule, la réception d'un important lot de pupes et l'adoption du lapin comme animal-hôte nous a permis d'obtenir un élevage florissant de cette espèce.

Malgré des incidents divers et, en particulier, une intoxication accidentelle par insecticide qui a faillı détruire cet élevage, les effectifs ont augmenté de façon remarquable et sont passés, 
en 450 jours, d'une moyenne de 45 femelles jour à plus de 2.300 femelles.

Les modifications apportées à la méthode d'élevage permettent de nourrir un grand nombre d'insectes dans un minimum de temps tout en obtenant des productions de pupes ou d'adultes excédentaires et susceptıbles d'être utilisés à des fins expérimentales.

Cette méthode pourrait être appliquée sans grandes modifications d̀ un élevage en masse en vue d'une production de mâles stériles.

\section{Remerciements.}

Nous adressons nos plus vifs remerciements au Dr J. GRUVEL, Chef du Service d'Entomologie au laboratore de FARCHA (Rép. du Tchad), qui a bien voulu récolter et nous expédier l'important lot de pupes ayant permis la constitution de notre élevage.

Institut d'Elevage et de Médecine Vétérinare des Pays Tropicaux. Laboratoire d'Entomologie. Maisons-Alfort.

\title{
SUMMARY
}

Observations on the breeding of Glossina tachinoides (West.) using the rabbit as host

The use of the rabbit as host, and the receipt of a large quantity of pupae have enabled $G l$. tachinoides to be bred in abundance since early 1967.

In spite of an accidental intoxication by insecticide which coused the numbers to fall to less than 400 females per day, after 6 months they had risen to 2.300 females. The maximum longevity of females was 210 days and the production of pupae reached 695 per 100 females.

The technique used is suitable for application to the mass production of sterile males.

\author{
RESUMEN \\ Observaciones sobre una crianza de Glossina tachlnoides West., \\ siendo elegido el conejo como animal-huesped
}

Desde el comienza del año 1967, la adopción del conejo como animal huesped y la recepción de numerosas pupas permitieron obtener una crianza próspera de Gl. tachinoides, A pesar de una intoxicación accidental causada por insecticida, que ha provocado la disminución del número de las glosinas hasta menos de 400 hembras/dia, el dicho excedia 2.300 hembras a los 6 meses. Fué de 210 días la longevidad maxima de las hembras y la producción de pupas llegó a 695 pupas por 100 hembras.

Se podria utilizar esta técnica para la producción de machos esteriles en una crianza extensiva.

\section{BIBLIOGRAPHIE}

AZEVEDO (J. Fraga de) et PINHAO (R. C.).Perspectives offerfes par l'élevage en laboratoire de Glossina morsitans d̀ Lisbonne. (Prospects offered by the laboratory breeding of Glossina morsitans in Lisbon). Panel on control of Livestock Insect Pests by the sterile-Male Technique. JointF.A.O.Jl.A.E.A., Vienne (Autriche), 23-27 janvier 1967.

BUXTON (P. A.) et LEWIS (D. J.). - Climat et 'mouches tsétsé : études en laboratoire, sur Gl. submorsitans ef tachinoides. (Climate and Tsetse flies : laboratory studies upon Gl. submorsitans and fachinoides). Philos. Trans. (B), 1934, $224: 175-240$.

ITARD (J.) et MAILLOT (L.). - Notes sur un élevage de Glossines (Diptera-Muscidae) entrepris, à partir de pupes expédiées d'Afrique, d̀ Maisons-Alfort (France). Rev. Elev. Méd. vét. Pays trop., 1966, 19 (1): 29-44. JORDAN (A. M.), NASH (T. A.M.) et BOYLE (J.) 

A.). - L'élevage de Glossino ousfeni Newst. sur les lapins à oreilles pendantes. I. Effi- cacité de la méthode. (The rearing of Glossina austeni Newst. with lop-eared rabbits as hosts. I. Efficacity of the method). Ann. Trop. Med. Porosit., 1967, 61 (2):182-188. NASH (T. A. M.), JORDAN (A. M.) et BOYLE (J. A.). - Une méthode prometteuse pour

élever Glossina austeni (Newst.) à petite échelle, basée sur l'utilisation du lapin à oreilles pendantes comme donneur de sang. (A promising method for rearing Glossino austeni (Newst.) on a small scale, based on the use of rabbits' ears for feeding). Trons. R. Soc, trop. Med. Hyg., 1966, 60 (2) : 183188. 